\title{
Medición del Flujo de Acceso utilizando los distintos sentidos de la punción arterial mediante técnica de dilución con ultrasonidos
}

\author{
Premio Izasa. Accesos Vasculares y Nuevas Tecnologías
}

\section{Gustavo Samuel Aguilar Gómez - Antonio José Fernández Jiménez - Irene Cabrera Plaza}

Centro de Hemodiálisis Sierra Este, Diálisis Andaluza

\section{Resumen}

La medición del flujo del acceso vascular con técnica de dilución con ultrasonidos está basada en estudios de Krivitski que aconsejan realizar la punción arterial en sentido proximal a la anastomosis, porque la mayor turbulencia mejora la dilución del suero fisiológico. Esto supone una limitación en el conocimiento del flujo de los accesos vasculares en los que no es posible 0 aconsejable realizar la punción arterial sentido proximal a la anastomosis.

Desarrollamos un estudio autocontrolado para detectar y cuantificar la posible diferencia entre las mediciones del flujo del acceso realizadas con punción arterial proximal y distal a la anastomosis mediante Transonic HDO1®; obteníamos 2 determinaciones del flujo en cada sentido de la punción arterial, correspondientes a las rotaciones opuestas del bisel de la aguja.

Encontramos diferencias estadísticamente significativas $(<0.05)$ entre el flujo con las punciones en sentido distinto; esta diferencia fue de 164,78 $\mathrm{ml} /$ min de media (intervalo de 55,63 a 273,93 $\mathrm{ml} / \mathrm{min}$ ) a favor del flujo obtenido con la punción arterial en sentido proximal a la anastomosis. No encontramos diferencias significativas entre los flujos hallados con distintas rotaciones de la aguja pero igual sentido de la punción.

\section{Correspondencia: \\ Gustavo Samuel Aguilar Gómez \\ Avd. Innovación n², Edif. Sierra Este. \\ 41020 Sevilla \\ gussagacupuntor@telefonica.net}

Por tanto, consideramos el sentido de punción de la aguja arterial como factor que condiciona la medición del flujo del acceso vascular mediante el método de dilución con ultrasonidos, pero conocida su variación, podemos incluir accesos vasculares que antes eran excluidos en la monitorización con Transonic@.

PALABRAS CLAVE:

- HEMODIÁLISIS

- ACCESO VASCULAR

- FLUJO SANGUÍNEO

- MEDIDORES DE CAUDAL

Measurement of Access Flow using the different directions of arterial puncture with ultrasound dilution technique

\section{Abstract}

Measuring vascular access flow using the ultrasound dilution technique is based on studies by Krivitski that advise puncturing the artery proximal to the anastomosis, because the greater turbulence improves the dilution of the saline. This limits knowledge about the flow of the vascular accesses where it is not possible or advisable to perform the arterial puncture proximal to the anastomosis.

We carried out a self-controlled study to detect and quantify the possible difference between access flow measurements in arterial puncture proximal and distal to the anastomosis using Transonic HDO1®; we obtained 2 flow determinations in each arterial puncture direction, corresponding to the opposite rotations of the needle bevel. 
We found statistically significant differences $(<0.05)$ between the flows with the punctures in different directions; the mean difference was $164.78 \mathrm{ml} / \mathrm{min}$ (interval of 55.63 to $273.93 \mathrm{ml} /$ min) in favour of the flow obtained with arterial puncture proximal to the anastomosis. We did not find significant differences between the flows observed with different rotations of the needle but in the same puncture direction.

Accordingly, we consider the direction of puncture as a factor that conditions the measurement of vascular access flow using the ultrasound dilution method, but knowing this variation we can include vascular accesses that were previously excluded in monitoring with Transonic $®$.

\section{KEY WORDS:}

HAEMODIALYSIS

VASCULAR ACCESS

BLOOD FLOW

FLOW MEASUREMENT DEVICES

\section{Introducción}

La supervivencia del acceso vascular (AV), y por tanto el pronóstico del paciente renal en hemodiálisis (HD), depende en gran medida de la vigilancia que se ejerza sobre él. Enfermería interviene de manera directa en este proceso utilizando entre otras técnicas y métodos de monitorización, la dilución con ultrasonidos, cuyos valores predicen de manera fiable posibles alteraciones en el funcionamiento del $\mathrm{AV}^{1}$, para detectar precozmente lesiones estructurales tratables con el objetivo de prevenir trombosis y aumentar la supervivencia del AV ${ }^{2}$.

En nuestro centro venimos desarrollando desde hace 9 años un programa de control y seguimiento del AV basado en las indicaciones de la Sociedad Española de Nefrología; como son la exploración física diaria, cambio en las presiones venosas y arteriales, etc ${ }^{2}$ y cuya piedra angular es la medición de la Recirculación (R) y del Flujo del Acceso (QA) mediante un medidor de caudal sanguíneo: monitor Transonic HD01®.

El método que empleamos para cuantificar estos parámetros es el descrito por Krivitski, consistente en rea- lizar inyecciones de $10 \mathrm{ml}$ de suero fisiológico por la rama venosa durante 4 ó 5 segundos con la ultrafiltración anulada y esperar al análisis del software específico de las diferencias dilucionales obtenidas entre las distintas ramas donde hemos colocado los detectores de Q-dilución, no siendo necesaria la anulación de la ultrafiltración para hallar el QA. Su autor aconseja realizar la punción arterial el día de la medición en sentido proximal a la anastomosis, en la mayoría de los casos en sentido distal respecto a la posición anatómica del brazo, ya que las condiciones de mezcla del indicador de suero fisiológico y de sangre son más favorables, por la existencia de mayor turbulencia al realizarse la infusión a contracorriente del flujo sanguíneo por estar las líneas invertidas cuando medimos el QA ${ }^{1}$. De esta manera asume que la punción arterial a favor de flujo no garantiza que la mezcla sea completa debido a la falta de turbulencias.

Debido a esto, nuestro protocolo incluye solamente el seguimiento de los AVs; fístulas autólogas y prótesis, a los que es posible la punción arterial en el sentido proximal a la anastomosis, entre otros requisitos. Esto supone una limitación en el conocimiento del QA de los AVs de pequeño desarrollo en longitud, profundos o dificultosos de puncionar en sentido proximal a la anastomosis, precisamente AVs complicados en los que tenemos el máximo interés en monitorizar sus flujos de acceso. Los accesos vasculares con estas características, por tanto, estaban excluidos de la monitorización del QA.

Sin embargo, en varias ocasiones hemos medido el $Q A$, realizando la medición con la punción arterial en sentido distal a la anastomosis o en sentido proximal respecto a la posición anatómica del brazo, para tener una medición de referencia del acceso. Pero realmente no sabemos el valor comparativo de estas mediciones respecto a las obtenidas con la punción arterial en sentido proximal a la anastomosis. De la necesidad de saber la variabilidad de las mediciones respecto a los distintos sentidos de punción arterial del acceso, cuando la punción arterial en sentido distal a la anastomosis no es posible, surge nuestro problema de estudio: si no existe diferencia podremos aplicar el protocolo con el sentido de la punción indistinto y por tanto desarrollar la monitorización de los QA de estos AVs complicados. Y si existe diferencia, cuantificarla, para considerar las mediciones que se hagan en estas circunstancias en el contexto adecuado. 


\section{Objetivos}

- Detectar si existe diferencia entre las mediciones del QA realizadas con punción arterial proximal y distal a la anastomosis mediante Transonic HDO1®.

- Cuantificar la posible diferencia o correlación entre ambas mediciones, para considerar en su caso la punción de la aguja arterial como factor que puede condicionar la medición del QA de un AV mediante Transonic ${ }^{\circledR}$.

- Contribuir con los resultados a la utilización más adecuada de nuestro protocolo.

Partimos de la hipótesis nula: "No existen diferencias significativas en las mediciones del QA con método de dilución con ultrasonidos, realizadas con punciones arteriales sentido proximal y sentido distal a la anastomosis".

\section{Material y Método}

Estudiamos una muestra de 34 pacientes procedentes de nuestro centro periférico de hemodiálisis Sierra Este de Sevilla durante 6 meses (octubre 2007marzo 2008).

Los criterios de inclusión para la obtención de la muestra de todos los pacientes del centro fueron:

- Fístula arteriovenosa madura o prótesis, normofuncionante y que pudiera usarse en bipunción.

- Trayecto lineal comunicado que permitiese ambas punciones, arterial y venosa, en la misma vena y distanciadas sus puntas $\geq 6 \mathrm{cms}$.

- $\quad$ El AV debía permitir la punción arterial a contracorriente al flujo sanguíneo.

Se solicitó y obtuvo el consentimiento informado por parte de los pacientes previamente a la realización de las mediciones.

Desarrollamos un estudio autocontrolado en el que realizamos a cada paciente una medición inicial de referencia de la recirculación y del flujo del acceso con la punción arterial en sentido proximal a la anastomosis $(\mathrm{ml})$ y posteriormente realizamos la segunda medición con la punción arterial en sentido distal a la anastomosis (m2); las dos mediciones se realizaron en sesiones contiguas, excluyendo el primer día de la semana, para evitar la situación de sobrecarga hídrica inmediatamente posterior al fin de semana y homogeneizar las condiciones de medición. La totalidad de las mediciones en sentido distal se realizaron con agujas de calibre $15 \mathrm{G}$, solo un caso en sentido proximal se realizó con ambas agujas de calibre 16G.

El material que utilizamos fue un ordenador portátil como soporte de registro y almacenamiento con software específico, un monitor de flujo (Q) Transonic $\mathrm{HDOl}{ }^{\circledR}$ con conexiones para el ordenador, sensores de $Q$ /dilución, jeringas con agujas, suero fisiológico, conductor de ultrasonidos y una hoja de registro manual en la que se recogieron los siguientes datos: nombre, fecha de realización, tensión arterial preestudio, tiempo de tratamiento transcurrido a la hora de realizar la prueba, tipo y localización de la fístula, recirculación con líneas normales $(R L N)$, con las líneas invertidas (RLI) y QA.

Las consideraciones previas que se tuvieron en cuenta inmediatamente antes de cada medición fueron la de asegurarnos la situación hemodinámica del paciente desarrollando la medición dentro de las dos primeras horas de sesión dialítica, aunque aceptamos alguna medida fuera de tiempo tras la comprobación correspondiente de la TA y las punciones adecuadas.

En las mediciones con la punción en sentido proximal de la anastomosis ( $\mathrm{ml}$ ) realizamos:

- Dos determinaciones de la recirculación, una con las líneas en posición normal y otra con las líneas en posición invertida (RLNml y RLIml)

- Dos determinaciones del flujo de acceso con inversión de líneas

- La primera con el bisel hacia abajo, referenciado por el punto rojo que la aguja muestra en su zona superior (QA PR)

- La segunda con el bisel hacia arriba, referenciado por el punto negro (QA PN). 
En las mediciones con la punción en sentido distal (m2) realizamos las determinaciones del mismo modo, es decir:

- Dos determinaciones de la recirculación, una con las líneas en posición normal y otra con las líneas en posición invertida (RLNm2 y RLIm2)

- Dos determinaciones del flujo de acceso con inversión de las líneas

- La primera con el bisel hacia abajo, referenciado por el punto rojo que la aguja muestra en su zona superior (QA PR2)

- La segunda con el bisel hacia arriba, referenciado por el punto negro (QA PN2).

Por tanto, al finalizar ambos tipos de mediciones teníamos 2 determinaciones del flujo en cada sentido de punción arterial.

Recopilamos los datos de las gráficas de HD y de las hojas de registro de mediciones, en una base de datos para su tratamiento con el paquete estadístico SPSS en su versión 16.0. Utilizamos el test estadístico de contrastación de normalidad de Kolmogorov-Smirnov, el test de comparación de medias T Student para datos apareados y el test de correlación paramétrico de Pearson y no paramétrico de Rho de Spearman.

\section{Resultados}

El $64,7 \%$ de la muestra fueron hombres y el $35,3 \%$ mujeres, con una edad media de 62,03 $\pm 16,54$ años. Sus accesos vasculares fueron 29 fístulas autólogas $(85,3 \%)$ y 5 protésicas (14,7\%). La distribución de las anastomosis de los AVs que formaron parte del estudio fue: radio-cefálica de Cimino-Brescia o de antebrazo $41 \%$, húmero-mediana $17,6 \%$, húmero-basílica $8,8 \%$, húmero-cefálica $20,6 \%$ y húmero-axilar $11,8 \%$.

En la tabla 1 se exponen los valores de las principales variables obtenidas en cada medición junto a la aclaración de sus abreviaturas. En las mediciones en sentido proximal de la anastomosis ( $\mathrm{ml}$ ) obtuvimos el $100 \%$ de las mediciones del flujo con el bisel hacia abajo frente al $79,4 \%$ de las mediciones del flujo con el bisel hacia arriba. En las mediciones en sentido distal (m2) obtuvimos también el $100 \%$ de las mediciones del flujo con el bisel hacia abajo frente al $73.5 \%$ de las mediciones del flujo con el bisel hacia arriba.

\begin{tabular}{|c|c|c|c|}
\hline & $\begin{array}{l}\text { Medición } \\
\text { con Punción } \\
\text { Arterial } \\
\text { sentido } \\
\text { proximal a la } \\
\text { anastomosis } \\
\text { (m1) }\end{array}$ & $\begin{array}{c}\text { Medición } \\
\text { con Punción } \\
\text { Arterial } \\
\text { sentido distal } \\
\text { a la } \\
\text { anastomosis } \\
\text { (m2) }\end{array}$ & unidades \\
\hline \multirow{2}{*}{$\begin{array}{c}\text { Recirculación } \\
\text { con líneas } \\
\text { en posición } \\
\text { normal }\end{array}$} & RLNml & RLNm2 & \\
\hline & 0 & 0 & $\begin{array}{c}\text { Porcentaje } \\
(\%)\end{array}$ \\
\hline \multirow{2}{*}{$\begin{array}{l}\text { Recirculación } \\
\text { con líneas } \\
\text { en posición } \\
\text { invertidas }\end{array}$} & RLIml & RLIm2 & \\
\hline & $22,88 \pm 11,15$ & $23,15 \pm 8,91$ & $\begin{array}{c}\text { Porcentaje } \\
(\%)\end{array}$ \\
\hline \multirow{2}{*}{$\begin{array}{l}\text { Flujo de } \\
\text { Acceso con } \\
\text { bisel abajo }\end{array}$} & QA PR & QA PR2 & \\
\hline & $\begin{array}{c}1265,88 \pm \\
815,11\end{array}$ & $\begin{array}{c}1112,94 \pm \\
694,76\end{array}$ & $\begin{array}{l}\text { Media } \pm \\
\mathrm{DE}(\mathrm{ml} / \\
\mathrm{min})\end{array}$ \\
\hline \multirow{2}{*}{$\begin{array}{l}\text { Flujo de } \\
\text { Acceso con } \\
\text { bisel arriba }\end{array}$} & QA PN & QA PN2 & \\
\hline & $\begin{array}{c}1233,33 \pm \\
796,45\end{array}$ & $\begin{array}{c}1154,80 \pm \\
682,49\end{array}$ & $\begin{array}{c}\text { Media } \pm \\
\mathrm{DE}(\mathrm{ml} / \\
\mathrm{min})\end{array}$ \\
\hline \multirow{2}{*}{$\begin{array}{l}\text { Media de } \\
\text { los Flujos } \\
\text { de Acceso } \\
\text { con distintas } \\
\text { posiciones } \\
\text { del bisel } \\
\text { pero mismo } \\
\text { sentido de } \\
\text { punción }\end{array}$} & QAml & QAm2 & \\
\hline & $\begin{array}{c}1243,33 \pm \\
809,74\end{array}$ & $\begin{array}{c}1152,60 \pm \\
691,55\end{array}$ & $\begin{array}{l}\text { Media } \pm \\
\text { DE (ml/ } \\
\mathrm{min})\end{array}$ \\
\hline
\end{tabular}

Tabla 1. Valores de las principales variables obtenidas en cada medición junto a la aclaración de sus abreviaturas

Distinguiendo los flujos por los tipos de acceso vascular y sus anastomosis, encontramos los resultados que se muestran en la tabla 2. Distinguiendo los flujos por rotaciones del bisel y sentidos de punción, encontramos los resultados que se exponen en la tabla 3.

Asumimos que las mediciones del flujo, sus diferencias medias entre distintas posiciones del bisel o sentido de punción arterial, y sus medias homogeneizando los resultados respecto al sentido de la punción arterial (QAm1 y QAm2), tenían distribución normal comprobada con el test estadístico de contraste de normalidad de Kolmogorov-Smirnov. Al realizar el test de Student para comparación de medias de datos apareados encontramos diferencias estadísticamente significativas $(<0,05)$, entre QA PR y QA PR2, entre QA PN y QA PN2 y entre QAml y QAm2. En la tabla 4 se muestran los estadísticos de las comparaciones significativas. No se encontraron sin embargo diferencias significativas entre el flujo con las distintas rotaciones de la aguja pero igual sentido de punción 
(las diferencias medias en $\mathrm{ml}$ entre QA PR y QA PN fueron de 20,00 $\pm 231,94 \mathrm{ml} / \mathrm{min}$, y en $\mathrm{m} 2$ entre $Q A$ PR2 y QA PN2 de $-4,40 \pm 256,92 \mathrm{ml} / \mathrm{min}$ ).

\begin{tabular}{|c|c|c|c|c|c|}
\hline $\begin{array}{c}\text { Tipo de } \\
\text { AV }\end{array}$ & \multicolumn{2}{|c|}{ Anastomosis } & QAml & QAm2 & QA \\
\hline \multirow{10}{*}{ Fístula } & \multirow{2}{*}{ Húmero-basílica } & $\begin{array}{l}\text { Media } \\
(\mathrm{ml} / \mathrm{min})\end{array}$ & 2125,00 & 1787,50 & 1956,20 \\
\hline & & $\mathrm{DE}$ & 799,03 & 795,49 & 797,26 \\
\hline & \multirow{2}{*}{ Humero-cefálica } & $\begin{array}{c}\text { Media } \\
(\mathrm{m} / \mathrm{min})\end{array}$ & 1204,00 & 994,00 & 1099,00 \\
\hline & & $\mathrm{DE}$ & 459,05 & 307,19 & 370,26 \\
\hline & \multirow{2}{*}{ Humero-mediana } & $\begin{array}{c}\text { Media } \\
(\mathrm{ml} / \mathrm{min})\end{array}$ & 1491,25 & 1675,00 & 2250,00 \\
\hline & & $\mathrm{DE}$ & 1629,13 & 1401,06 & 1912,72 \\
\hline & \multirow{2}{*}{$\begin{array}{c}\text { Radio-cefalica } 0 \\
\text { de Cimino-Brescia }\end{array}$} & $\begin{array}{c}\text { Media } \\
(\mathrm{ml} / \mathrm{min})\end{array}$ & 1052,27 & 1033,18 & 1072,50 \\
\hline & & $D E$ & 641,90 & 573,78 & 609,99 \\
\hline & \multirow{2}{*}{ Total } & $\begin{array}{l}\text { Media } \\
(\mathrm{ml} / \mathrm{min})\end{array}$ & 1264,09 & 1187,38 & 1296,44 \\
\hline & & $\mathrm{DE}$ & 866,42 & 711,00 & 801,97 \\
\hline \multirow{6}{*}{ Prótesis } & \multirow{2}{*}{ Húmero-axilar } & $\begin{array}{l}\text { Media } \\
(\mathrm{m} / \mathrm{min})\end{array}$ & 1197,50 & 973,33 & 1100,83 \\
\hline & & $\mathrm{DE}$ & 630,00 & 777,32 & 772,35 \\
\hline & \multirow[t]{2}{*}{ Húmero-basílica } & $\begin{array}{c}\text { Media } \\
(\mathrm{m} / \mathrm{min})\end{array}$ & 970,00 & 960,00 & 965,00 \\
\hline & & DE & . & . & . \\
\hline & \multirow[t]{2}{*}{ Total } & $\begin{array}{c}\text { Media } \\
(\mathrm{m} / \mathrm{min})\end{array}$ & 1152,00 & 970,00 & 1066,87 \\
\hline & & $\mathrm{DE}$ & 555,00 & 634,71 & 634,26 \\
\hline \multirow{12}{*}{ Total } & \multirow{2}{*}{ Húmero-axilar } & $\begin{array}{l}\text { Media } \\
(\mathrm{m} / \mathrm{min})\end{array}$ & 1197,50 & 973,33 & 1100,83 \\
\hline & & $\mathrm{DE}$ & 630,00 & 777,32 & 772,35 \\
\hline & \multirow[t]{2}{*}{ Húmero-basílica } & $\begin{array}{c}\text { Media } \\
(\mathrm{m} / \mathrm{min})\end{array}$ & 1740,00 & 1511,66 & 1625,83 \\
\hline & & $\mathrm{DE}$ & 874,01 & 738,00 & 803,33 \\
\hline & \multirow{2}{*}{ Humero-cefálica } & $\begin{array}{c}\text { Media } \\
(\mathrm{m} / / \mathrm{min})\end{array}$ & 1204,00 & 994,00 & 1099,00 \\
\hline & & $\mathrm{DE}$ & 459,05 & 307,19 & 370,26 \\
\hline & \multirow{2}{*}{ Humero-mediana } & $\begin{array}{c}\text { Media } \\
(\mathrm{m} / / \mathrm{min})\end{array}$ & 1491,25 & 1675,00 & 2250,00 \\
\hline & & $\mathrm{DE}$ & 1629,13 & 1401,06 & 1912,72 \\
\hline & \multirow{2}{*}{$\begin{array}{c}\text { Radio-cefalica } 0 \\
\text { de Cimino-Brescia }\end{array}$} & $\begin{array}{c}\text { Media } \\
(\mathrm{m} / \mathrm{min})\end{array}$ & 1052,27 & 1033,18 & 1072,50 \\
\hline & & $\mathrm{DE}$ & 641,90 & 573,78 & 609,99 \\
\hline & \multirow{2}{*}{ Total } & $\begin{array}{l}\text { Media } \\
(\mathrm{m} / \mathrm{min})\end{array}$ & 1243,33 & 1152,60 & 1256,52 \\
\hline & & $\mathrm{DE}$ & 809,74 & 691,55 & 767,46 \\
\hline
\end{tabular}

QAml: Flujo de acceso con punción arterial en sentido proximal a la anastomosis

QAm2: Flujo de acceso con punción arterial en sentido distal a la anastomosis

Tabla 2. Flujos según tipos de acceso vascular y sus anastomosis

\begin{tabular}{|c|c|c|c|c|c|}
\hline \multicolumn{2}{|c|}{$\begin{array}{c}\text { Tipo de acceso } \\
\text { vascular }\end{array}$} & \multirow{2}{*}{$\frac{\text { QA PR }}{29}$} & \multirow{2}{*}{$\frac{\text { QA PN }}{22}$} & \multirow{2}{*}{$\begin{array}{c}\text { QA PR2 } \\
29\end{array}$} & \multirow{2}{*}{$\begin{array}{c}\text { QA PN2 } \\
21\end{array}$} \\
\hline \multirow{4}{*}{ Fístula } & $N$ & & & & \\
\hline & $\begin{array}{c}\text { Media } \\
(\mathrm{ml} / \mathrm{min})\end{array}$ & 1283,10 & 1255,00 & 1141,38 & 1171,43 \\
\hline & $\mathrm{DE}$ & 850,286 & 859,700 & 714,366 & 702,675 \\
\hline & $\begin{array}{c}\% \text { del } \\
\text { Total N }\end{array}$ & $85,3 \%$ & $81,5 \%$ & $85,3 \%$ & $84,0 \%$ \\
\hline \multirow{4}{*}{ Prótesis } & $N$ & 5 & 5 & 5 & 4 \\
\hline & $\begin{array}{c}\text { Media } \\
(\mathrm{ml} / \mathrm{min})\end{array}$ & 1166,00 & 1138,00 & 948,00 & 1067,50 \\
\hline & $\mathrm{DE}$ & 637,048 & 478,613 & 608,375 & 650,045 \\
\hline & $\begin{array}{l}\% \text { del } \\
\text { Total N }\end{array}$ & $14,7 \%$ & $18,5 \%$ & $14,7 \%$ & $16,0 \%$ \\
\hline \multirow{4}{*}{ Total } & $N$ & 34 & 27 & 34 & 25 \\
\hline & $\begin{array}{c}\text { Media } \\
(\mathrm{ml} / \mathrm{min})\end{array}$ & 1265,88 & 1233,33 & 1112,94 & 1154,80 \\
\hline & $\mathrm{DE}$ & 815,112 & 796,454 & 694,762 & 682,490 \\
\hline & $\begin{array}{l}\text { \% del } \\
\text { Total N }\end{array}$ & $100,0 \%$ & $100,0 \%$ & $100,0 \%$ & $100,0 \%$ \\
\hline
\end{tabular}

QA PR: Flujo de acceso con bisel hacia abajo y punción en sentido proximal a la anastomosis

QA PN: Flujo de acceso con bisel hacia arriba y punción en sentido proximal a la anastomosis

QA PR2: Flujo de acceso con bisel hacia abajo y punción en sentido distal a la anastomosis

QA PN2: Flujo de acceso con bisel hacia arriba y punción en sentido distal a la anastomosis

Tabla 3. Flujos según posición del bisel y sentidos de punción

La correlación gráfica entre los flujos obtenidos con la misma posición del bisel pero distinto sentido de punción se muestran en las figuras 1 y 2 . Las correlaciones fueron estadísticamente significativas de forma bilateral $(<0,01)$ con el test paramétrico de Pearson en todos los cruces del flujo con los distintos sentidos de punción arterial (proximal y distal) y con las distintas posiciones del bisel. Del mismo modo desarrollando el test no paramétrico de Rho de Spearman encontramos significación estadística bilateral $(<0,01)$ entre las mismas asociaciones: ambos tests mostraron correlación muy alta entre QAml y QAm2: $(0,958$ y 0,881 respectivamente, ambos con un nivel de significación bilateral del 0,01.

\section{Discusión}

No se hallaron diferencias significativas entre las recirculaciones con las punciones realizadas en sentido distal o proximal a la anastomosis; se ha de tener en cuenta que la recirculación con líneas invertidas (RLI) es un indicador de control de la realización de la prueba y su variabilidad no hubiera tenido relevancia clínica. 


\begin{tabular}{|c|c|c|c|c|c|c|c|}
\hline \multicolumn{8}{|c|}{ Test de T-Student para datos apareados } \\
\hline & \multirow{2}{*}{$\begin{array}{c}\text { Media } \\
(\mathrm{ml} / \mathrm{min})\end{array}$} & \multirow{2}{*}{ DE } & \multicolumn{2}{|c|}{$95 \%$ Intervalo de confianza } & \multirow{2}{*}{$\mathrm{T}$} & \multirow{2}{*}{$\begin{array}{c}\text { grados } \\
\text { de libertad }\end{array}$} & \multirow{2}{*}{ Sig.bilateral } \\
\hline & & & $\begin{array}{l}\text { límite } \\
\text { inferior }\end{array}$ & $\begin{array}{l}\text { límite } \\
\text { superior }\end{array}$ & & & \\
\hline QA PR - QA PR2 & 152,941 & 330,386 & 37,664 & 268,218 & 2,699 & 33 & 011 \\
\hline QA PN - QA PN2 & 144,783 & 267,001 & 29,323 & 260,243 & 2,601 & 22 & 016 \\
\hline QAm1 - QAm2 & 164,78261 & 252,40875 & 55,63288 & 273,93234 & 3,131 & 22 & ,005 \\
\hline QAm2 - QAm1 & $-164,78261$ & 252,40875 & $-273,93234$ & $-55,63288$ & $-3,131$ & 22 &, 005 \\
\hline
\end{tabular}

QA PR: Flujo de acceso con bisel hacia abajo y punción en sentido proximal a la anastomosis

QA PN: Flujo de acceso con bisel hacia arriba y punción en sentido proximal a la anastomosis

QA PR2: Flujo de acceso con bisel hacia abajo y punción en sentido distal a la anastomosis

QA PN2: Flujo de acceso con bisel hacia arriba y punción en sentido distal a la anastomosis

QAml: Flujo de acceso con punción arterial en sentido proximal a la anastomosis

QAm2: Flujo de acceso con punción arterial en sentido distal a la anastomosis

Tabla 4. Resultados del Test de T-Student

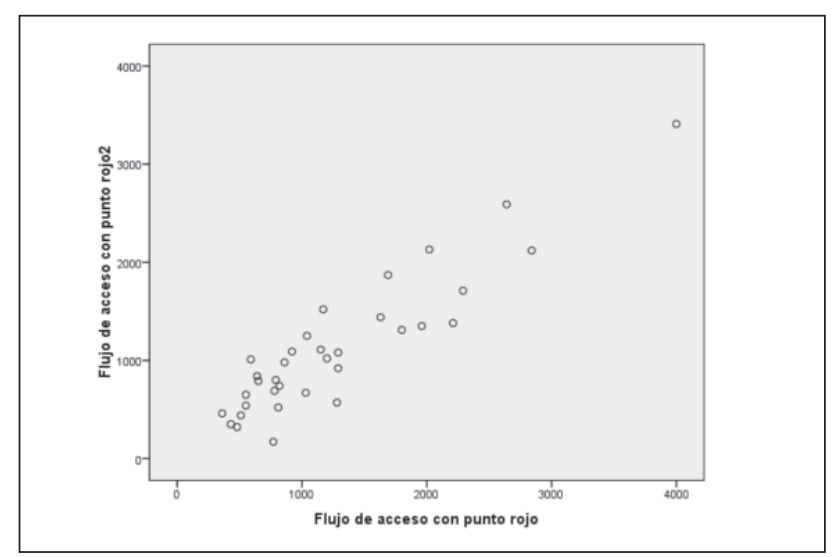

Figura 1. Correlación entre los flujos obtenidos con el bisel hacia abajo y distinto sentido de punción

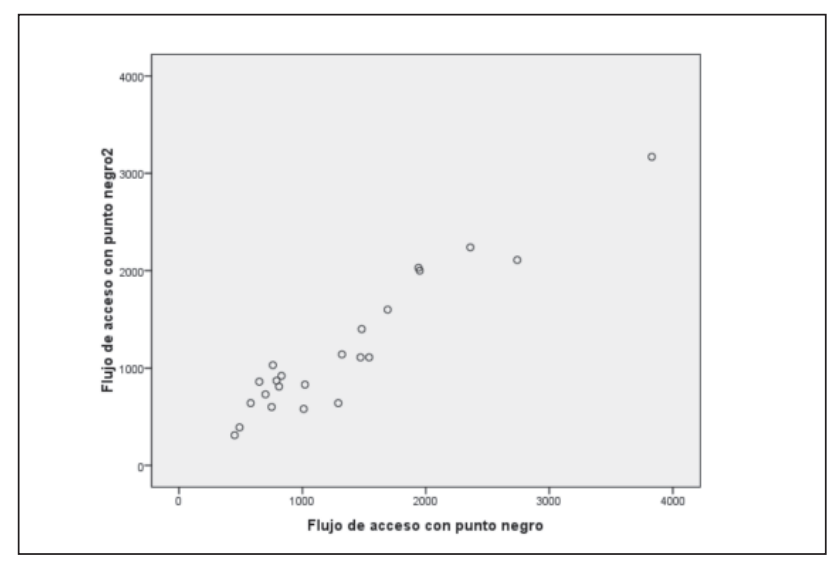

Figura 2. Correlación entre los flujos con el bisel hacia arriba y distinto sentido de punción
Las diferencias estadísticamente significativas entre las mediciones del flujo con la misma posición del bisel de la aguja y entre las medias obtenidas con diferentes posiciones del bisel pero igual sentido de punción, nos muestran de tres maneras distintas, la variabilidad que produce el sentido de la punción en el resultado de la medición del flujo con técnica de dilución con ultrasonidos.

Asumiendo estas diferencias como válidas, podemos decir que la variabilidad obtenida al medir el flujo con punciones arteriales en sentidos distintos a la anastomosis estará comprendida entre 55,63 y $273,93 \mathrm{ml} /$ $\mathrm{min}$, con un media de $164,78 \mathrm{ml} / \mathrm{min}$. Esta diferencia será a favor de la medición del flujo realizada con la punción en sentido proximal a la anastomosis, es decir, los valores que se obtendrán al realizar la prueba con la aguja a favor de flujo tenderán a ser menores que los obtenidos en sentido contracorriente.

Rechazamos pues la hipótesis nula inicial aceptando la hipótesis alternativa, y consideramos el sentido de la punción de la aguja arterial como factor que condiciona la medición del flujo de un acceso vascular mediante técnica de dilución con ultrasonidos. Y conocida su variación, debemos de incluir en el protocolo de monitorización con Transonic HDO1® la realización de la medición con la punción arterial en sentido distal a la anastomosis, ampliando de este modo la monitorización de las fístulas que por su dificultad 
eran excluidos del seguimiento, debiendo aclarar en la hoja de registro de Enfermería el sentido de punción en el que se realiza la prueba.

\section{Bibliografía}

1. Krivitski NM. Novel method to measure access flow during hemodialysis by ultrasound velocity dilution tecnique. ASAIO J41: M 741-M 745.1995.

2. Varios autores. Guía de acceso vascular en hemodiálisis. SEN. 2004.

3. Crespo R, Rivero F, Contreras MD, Martínez A, Fuentes MI. Pain degree and skin damage during arterio-venous fistula puncture. EDTNA ERCA J. 2004; 30(4):208-12.

4. NFK-DOQI Clinical practice guidelines for vascular access. Am J Kidney 1997; 30: S152-S191.
5. Agharazii M, Clouatre $Y$, Nolin L, Leblanc M. Variation of intra-access flow early and late into hemodialysis. ASAIO J. 2000; 46(4):452-5.

6. Krivitski NM. Theory and validation of access flow measurement by dilution technique during hemodialysis. Kidney Int. 1995; 48(1):244-50.

7. Krivitski NM, MacGibbon D, Gleed RD, Dobson A. Accuracy of dilution techniques for access flow measurement during hemodialysis. Am J Kidney Dis. 1998; 31(3):502-8.

8. McDonald JT, Sosa MA, Krivitski NM, Glidden D, Sands JJ. Identifying a new reality: zero vascular access recirculation using ultrasound dilution. ANNA J. 1996; 23(6):603-8.

9. Sands J, Glidden D, Jacavage W, Jones B. Difference between delivered and prescribed blood flow in hemodialysis. ASAIO J. 1996; 42(5):M717-9. 